\title{
Population-Level Effect of Cholera Vaccine on Displaced Populations, South Sudan, 2014
}

\author{
Andrew S. Azman, John Rumunu, \\ Abdinasir Abubakar, Haley West, Iza Ciglenecki, \\ Trina Helderman, Joseph Francis Wamala, \\ Olimpia de la Rosa Vázquez, William Perea, \\ David A. Sack, Dominique Legros, \\ Stephen Martin, Justin Lessler, \\ Francisco J. Luquero
}

Following mass population displacements in South Sudan, preventive cholera vaccination campaigns were conducted in displaced persons camps before a 2014 cholera outbreak. We compare cholera transmission in vaccinated and unvaccinated areas and show vaccination likely halted transmission within vaccinated areas, illustrating the potential for oral cholera vaccine to stop cholera transmission in vulnerable populations.

$\mathrm{I}$ n December 2013, violence erupted in Juba, South Sudan, and quickly spread throughout the country. By the end of 2014, one in five persons within the country had been displaced, and many sought refuge in protection of civilians (PoC) sites inside United Nations (UN) Mission bases and in spontaneous internally displaced persons (IDP) settlements. Within 6 weeks of the start of the violence, South Sudan Ministry of Health requested vaccine from the global oral cholera vaccine stockpile to target 163,000 IDPs in 6 camps throughout the country, but not persons in the broader host communities (1).

In April 2014, two months after vaccine deployment, South Sudan confirmed the first case of cholera in the country since $2009 ; \approx 4$ weeks later, officials declared a cholera outbreak. Over 5 months, 6,269 suspected cholera cases were reported, including 156 deaths. Most cases occurred outside vaccinated camps, often in communities or camps surrounding vaccinated populations.

Author affiliations: Johns Hopkins Bloomberg School of Public Health, Baltimore, Maryland, USA (A.S. Azman, D.A. Sack,

J. Lessler, F.J. Luquero); Ministry of Health, Juba, South Sudan

(J. Rumunu); World Health Organization, Juba (A. Abubakar,

J.F. Wamala); International Organization for Migration, Juba

(H. West); Médecins Sans Frontières, Geneva, Switzerland

(I. Ciglenecki); Medair, Ecublens, Switzerland (T. Helderman);

Médecins Sans Frontières, Barcelona, Spain (O. de la Rosa

Vázquez); World Health Organization, Geneva (W. Perea,

D. Legros, S. Martin); Epicentre, Paris, France (F.J. Luquero)

DOI: http://dx.doi.org/10.3201/eid2206.151592
Several studies have demonstrated the individual-level (direct) effects of oral cholera vaccination (2-4), but few have estimated the overall population-level effect (a combination of direct and indirect effects), which is critical to determining costs and benefits. To estimate the overall effect, the observed epidemic in vaccinated areas must be compared with a counterfactual epidemic that is modeled or based on an observed suitable control population.

We used detailed epidemiologic data from the 2014 vaccination campaigns and the subsequent cholera outbreak in South Sudan to determine how vaccine use may have altered the epidemic course in vaccinated areas. We compared epidemics in 2 areas that included vaccinated and unvaccinated populations: 1) PoC sites (vaccinated) and the community (unvaccinated) in Juba; and 2) Malakal PoC (vaccinated) and Wau Shilluk IDP (unvaccinated), 2 similar camps separated by a river.

\section{The Study}

The South Sudan Ministry of Health and World Health Organization implemented a clinic-based cholera surveillance system that captured basic patient data, laboratory results (if available), and outcomes. A suspected cholera case-patient was defined as anyone with acute watery diarrhea (diagnosed by a clinician); suspected cases were considered confirmed if the patient had a culture-positive fecal sample. Our analyses include all suspected cases.

We considered 5 populations in our comparisons, 3 in Juba County and 2 in Malakal County. In Juba, displaced persons were largely confined to 2 camps: 1) Tongping PoC camp (population 14,015) near the center of Juba; and 2) the UN House PoC camp (population 17,627) on the outskirts the city. We assumed all camp occupants were at risk for cholera and that, in the Juba community, only those residents without access to improved sanitation were at risk $(5,6)$ (online Technical Appendix, http://wwwnc.cdc.gov/ EID/article/22/6/15-1592-Techapp1.pdf).

Two-dose vaccine coverage among those eligible for vaccination (based on age and pregnancy status) was 93\% in Tongping $\mathrm{PoC}$ and $95 \%$ at UN House; the remaining Juba population was not vaccinated (1). In Malakal, we compared an informal unvaccinated IDP settlement, Wau Shilluk (population 39,000; online Technical Appendix), with an official PoC site, Malakal PoC camp (population 17,000; online Technical Appendix). Two-dose vaccine coverage in Malakal was $92.2 \%$ based on a coverage survey using systematic random sampling (1). 
Table. Effect of oral cholera vaccine by location, South Sudan, 2014*

\begin{tabular}{|c|c|c|c|c|c|}
\hline \multirow[b]{2}{*}{ Variable } & \multicolumn{5}{|c|}{ Location } \\
\hline & Juba† & Tongping & UN House & Wau Shilluk† & Malakal \\
\hline Setting type & Community & PoC Camp & PoC Camp & IDP camp & PoC camp \\
\hline Population vaccinated & No & Yes & Yes & No & Yes \\
\hline Population at risk & 387,512 & 14,015 & 17,627 & 39,000 & 17,000 \\
\hline No. cases $/ 10,000$ persons & 53.4 & 51.3 & 48.8 & 236.4 & 38.8 \\
\hline No. cases $/ 10,000$ children $<5$ y of age & 56.0 & 186.5 & 146.5 & - & - \\
\hline $\begin{array}{l}\text { Risk ratio, children }<5 \text { y compared with } \\
\text { those } \geq 5 \text { y of age }\end{array}$ & 1.0 & 3.6 & 3.0 & - & - \\
\hline No. days with $\mathrm{R}_{t}>1$ & $16 \ddagger$ & $2 \ddagger$ & $2 \ddagger$ & $14 \ddagger$ & $2 \ddagger$ \\
\hline Maximum $\mathrm{R}_{t}$ & 2.4 & 1.5 & 1.5 & 2.2 & 1.9 \\
\hline
\end{tabular}

We estimated the time-varying reproductive number of cholera within each location (online Technical Appendix) (7). We assumed that the median generation time for cholera followed a gamma distribution with a median of 5 days and that all infectious cases were clinically apparent. We calculated $95 \%$ CIs by using a multiple imputation and bootstrapping routine, in which we first stochastically imputed missing or inconsistent symptom onset times and then resampled observations with replacement (online Technical Appendix).

The cholera attack rate in the Juba community was 53.4 cases/10,000 persons at risk (i.e., 2,229 cases/387,512 persons at risk), compared with 49.9 cases $/ 10,000$ persons at risk in the Juba camps (i.e., 158 cases $/ 31,642$ persons at risk). Although the overall attack rates were similar, the age distribution in camps differed markedly from those in the community. In the community, the risk for cholera among children $<5$ and those $\geq 5$ years of age was nearly identical (risk ratio [RR] 1.0), but in the camps, the risk was substantially higher among children $<5$ years of age (Table; Figure 1). These age-specific differences in attack rates between camps and the community did not appear to be explained by population structure, age-specific vaccination coverage, or circulation of another diarrheal pathogen in the camps (online Technical Appendix); the differences point toward possible lower vaccine effectiveness among young children.

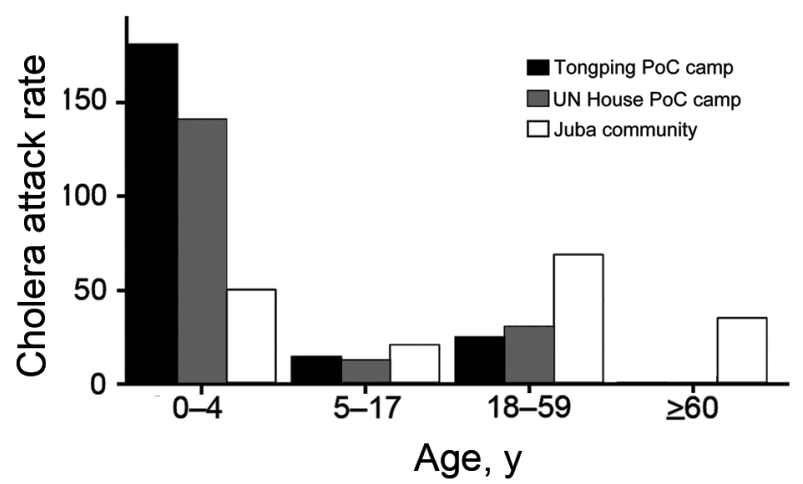

Figure 1. Estimated age-specific cholera attack rates (per 100,000 population) at different locations in Juba, South Sudan, 2014. PoC, protection of civilians; UN, United Nations.
The response mounted to oral vaccines is weaker in children than adults (8), although considerable uncertainty remains regarding the response to the oral cholera vaccine.

The difference in the estimated cumulative cholera attack rates between the unvaccinated Wau Shilluk IDP camp (236.4 cases/10,000 persons at risk) and the vaccinated Malakal PoC camp (38.8 cases/10,000 persons at risk) was even more striking (incidence rate ratio 6.1) (Table). Age-specific population figures were unavailable for Wau Shilluk.

Although differences in attack rates suggest a likely reduction in cholera risk in vaccinated areas and the possibility of age-dependent vaccine protection, these estimates are uncertain and should be cautiously interpreted. An alternative approach to understanding the effect of vaccination is to compare observed cholera transmission dynamics within vaccinated and unvaccinated populations.

The epidemic curves within vaccinated camps in Juba had no distinct peak and suggest a series of cholera introductions with little to no onward transmission (Figure 2). We estimated that the daily reproductive number $\left(\mathrm{R}_{t}\right.$; i.e., average number of secondary cases from a case becoming symptomatic on day $t$; online Technical Appendix) in vaccinated camps was $\leq 1$ for most of the epidemic. Each vaccinated camp had only 2 days on which the $95 \%$ CI of $\mathrm{R}_{t}$ was above unity. This finding contrasts with our estimates in unvaccinated areas, where despite conditions that may have been less suitable for transmission, $\mathrm{R}_{t}$ remained $>1$ for a sufficient and significantly longer time for an epidemic to progress ( $p<0.0001$; Table; online Technical Appendix).

\section{Conclusions}

We show that cholera vaccination campaigns likely played a key role in curtailing cholera transmission in vaccinated areas within South Sudan. The age-specific transmission patterns within the vaccinated camps in Juba suggest that vaccinated young children were less protected in the camps, although further investigation is needed to explore this and other possible explanations, including age-specific differences in care-seeking behavior between populations. 

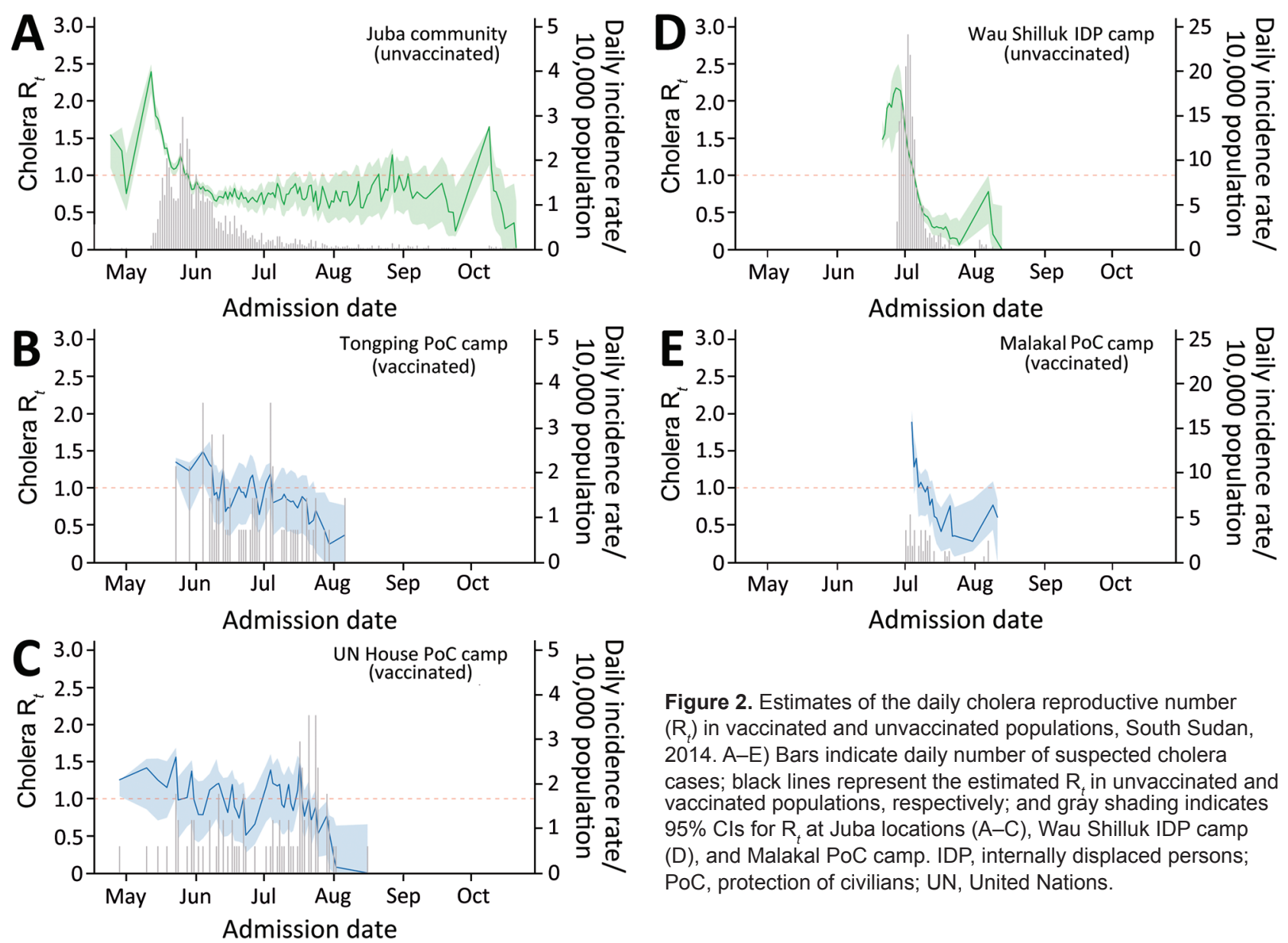

Figure 2. Estimates of the daily cholera reproductive number $\left(R_{t}\right)$ in vaccinated and unvaccinated populations, South Sudan, 2014. A-E) Bars indicate daily number of suspected cholera cases; black lines represent the estimated $R_{t}$ in unvaccinated and vaccinated populations, respectively; and gray shading indicates 95\% Cls for $\mathrm{R}_{t}$ at Juba locations (A-C), Wau Shilluk IDP camp (D), and Malakal PoC camp. IDP, internally displaced persons; PoC, protection of civilians; UN, United Nations.

Our study had several limitations. Analyses were based on suspected cases, which were defined by using a sensitive, but less specific, case definition; thus, many included cases were likely to be false positives. Our estimates of cholera attack rates depended on estimates of the population at risk in each area. We used the most reliable and up-to-date sources from agencies with an operational presence on the ground; however, the sizes of the dynamic community and camp populations used in the analyses were uncertain, and this uncertainty was not accounted for in the models. Last, we estimated the time-varying reproductive number of cholera by assuming a fixed generation time throughout the epidemic, which may not reflect reality due to the possibility of differences in care-seeking behavior and differential contraction of generation intervals between populations with an increasing prevalence of cholera (9).

Our findings provide evidence of the populationlevel effects of oral cholera vaccine. More work is needed to quantify this effect across multiple settings in reactive and preemptive deployments of the vaccine. High-quality surveillance and capacity to confirm suspect cases can greatly improve the possibility of making future estimates.

\section{Acknowledgments}

We acknowledge the work of all the public health workers involved in these vaccination campaigns and the larger cholera response, including members of the South Sudan National Cholera Task Force.

The work of A.S.A., D.A.S., J.L., and F.J.L. was supported by the Bill and Melinda Gates Foundation (OPP1089243 and the DOVE [Delivering Oral Vaccine Effectively] project, OPP153556).

Dr. Azman is a research associate in the Epidemiology Department at Johns Hopkins University Bloomberg School of Public Health. His research interests include infectious disease dynamics and vaccine study design, with a particular focus on cholera and hepatitis E.

\section{References}

1. Abubakar A, Azman AS, Rumunu J, Ciglenecki I, Helderman T, West H, et al. The first use of the global oral cholera vaccine emergency stockpile: lessons from South Sudan. 
PLoS Med. 2015;12:e1001901. http://dx.doi.org/10.1371/ journal.pmed.1001901

2. Luquero FJ, Grout L, Ciglenecki I, Sakoba K, Traore B, Heile M, et al. Use of Vibrio cholerae vaccine in an outbreak in Guinea. N Engl J Med. 2014;370:2111-20. http://dx.doi.org/10.1056/ NEJMoa1312680

3. Bhattacharya SK, Sur D, Ali M, Kanungo S, You YA, Manna B, et al. 5 year efficacy of a bivalent killed whole-cell oral cholera vaccine in Kolkata, India: a cluster-randomised, double-blind, placebo-controlled trial. Lancet Infect Dis. 2013;13:1050-6. http://dx.doi.org/10.1016/S1473-3099(13)70273-1

4. Ivers LC, Hilaire IJ, Teng JE, Almazor CP, Jerome JG, Ternier R, et al. Effectiveness of reactive oral cholera vaccination in rural Haiti: a case-control study and bias-indicator analysis. Lancet Glob Health. 2015;3:e162-8. http://dx.doi.org/10.1016/ S2214-109X(14)70368-7

5. Ali M, Lopez AL, You YA, Kim YE, Sah B, Maskery B, et al. The global burden of cholera. Bull World Health Organ. 2012;90:209-218A. http://dx.doi.org/10.2471/BLT.11.093427
6. World Health Organization/UNICEF Joint Monitoring Programme for Water Supply and Sanitation. Progress on drinking water and sanitation-2014 update. Geneva: World Health Organization; 2014

7. Wallinga J, Teunis P. Different epidemic curves for severe acute respiratory syndrome reveal similar impacts of control measures. Am J Epidemiol. 2004;160:509-16. http://dx.doi.org/10.1093/aje/ kwh255

8. Qadri F, Bhuiyan TR, Sack DA, Svennerholm A-M. Immune responses and protection in children in developing countries induced by oral vaccines. Vaccine. 2013;31:452-60. http://dx.doi.org/10.1016/j.vaccine.2012.11.012

9. Kenah E, Lipsitch M, Robins JM. Generation interval contraction and epidemic data analysis. Math Biosci. 2008;213:71-9. http://dx.doi.org/10.1016/j.mbs.2008.02.007

Address for correspondence: Andrew S. Azman, Johns Hopkins Bloomberg School of Public Health, 615 N Wolfe St, Rm E6003, Baltimore, MD 21205, USA; email: azman@jhu.edu

\section{April 2016: Food Safety Including:}

- Determinants and Drivers of Infectious Disease Threat Events in Europe

- Shiga Toxin-Producing Escherichia coli O157, England and Wales, 1983-2012

- Nosocomial Co-Transmission of Avian Influenza A(H7N9) and A(H1N1)pdm09 Viruses between 2 Patients with Hematologic Disorders

- Quantifying Transmission of Clostridium difficile within and outside Healthcare Settings

- Microevolution of Monophasic Salmonella Typhimurium during Epidemic, United Kingdom, 2005-2010

- Molecular Typing and Epidemiology of Human Listeriosis Cases, Denmark, 2002-2012

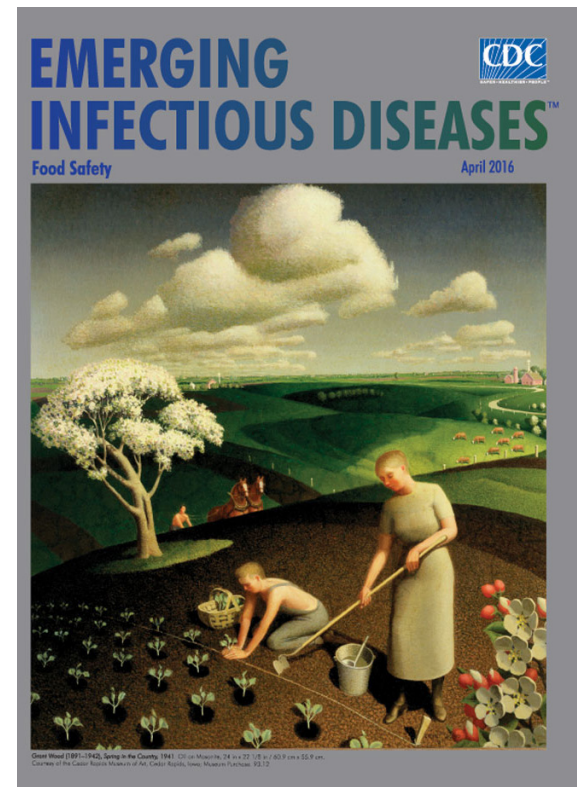

- Limited Dissemination of Extended-Spectrum $\beta$-Lactamaseand Plasmid-Encoded AmpC-Producing Escherichia coli from Food and Farm Animals, Sweden

- Post-Ebola Syndrome, Sierra Leone

- Transmission of Middle East Respiratory Syndrome Coronavirus Infections in Healthcare Settings, Abu Dhabi

- Lassa Virus Seroprevalence in Sibirilia Commune, Bougouni District, Southern Mali

- Nipah Virus Transmission from Bats to Humans Associated with Drinking Traditional Liquor Made from Date Palm Sap, Bangladesh, 2011-2014

- Evaluation of Viremia Frequencies of a Novel Human Pegivirus by Using Bioinformatic Screening and PCR

- Shiga Toxin 1-Producing Shigella sonnei Infections, California, United States, 2014-2015

http://wwwnc.cdc.gov/eid/articles/issue/22/04/table-of-contents 